\title{
Role of peripheral pan-retinal photocoagulation in diabetic macular edema treated with intravitreal ziv-aflibercept
}

This article was published in the following Dove Medical Press journal:

Clinical Ophthalmology

\author{
Ahmad M Mansour ${ }^{1,2}$ \\ Khalil El Jawhari ${ }^{3}$ \\ J Fernando Arevalo ${ }^{4}$ \\ 'Department of Ophthalmology, \\ American University of Beirut, \\ Beirut, Lebanon; ${ }^{2}$ Department \\ of Ophthalmology, Rafik Hariri \\ University Hospital, Beirut, Lebanon; \\ ${ }^{3}$ Medical School, Medical University \\ of Lodz, Lodz, Poland; ${ }^{4}$ Wilmer Eye \\ Institute, Johns Hopkins University \\ School of Medicine, Baltimore, \\ MD, USA
}

Purpose: The aim of this study was to investigate the possibility of decreasing the number of intravitreal anti-VEGF by peripheral pan-retinal photocoagulation (PPRP) in managing diabetic macular edema (DME) in a subcategory of patients who cannot comply to strict anti-VEGF follow-up protocols.

Materials and methods: This is a pilot prospective study. Consecutive patients with naïve DME were offered the choice of treatment and extend intravitreal ziv-aflibercept if they showed willingness for good compliance or PPRP with modified pro re nata intravitreal injections.

Results: Six eyes of 3 patients had PPRP versus 4 eyes of 4 patients had injections only. The number of anti-VEGF injections was markedly decreased when PPRP was administered from a mean number of injections of 16.8 (range 13-21; mean follow-up 24.3 months) to a mean number of 4.5 (range $0-8$; mean follow-up 33.7 months). Mean initial central macular thickness (CMT) was $462.0 \mu \mathrm{m}$ in the injection only group vs $457.3 \mu \mathrm{m}$ in the PPRP group. Mean final CMT was 462.0 in the injection only group vs 350.0 in the PPRP group. Baseline and final mean $\log$ MAR (Snellen equivalent) best-corrected visual acuity was initially and finally 0.84 $(20 / 137)$ and $0.60(20 / 80)$ in the injection only group and $0.70(20 / 100)$ and $0.69(20 / 98)$ in the PPRP group, respectively. The monthly cost for the PPRP group was one-third of the monthly cost for the injection only group.

Conclusion: PPRP allowed for a decrease in the number of intravitreal anti-VEGF injections in selected DME patients (sick, difficult to ambulate, financial burden, and fear of injections).

Keywords: vascular endothelial growth factor, laser therapy, diabetic retinopathy

\section{Introduction}

Diabetic macular edema (DME) being a major cause of visual impairment was managed in the past by focal laser photocoagulation and more recently by intravitreal injections of anti-vascular endothelial growth factor (VEGF) agents ${ }^{1}$ and less often by intravitreal dexamethasone implant or intravitreal corticosteroid injections. Despite an overwhelming evidence supporting anti-VEGF therapy for DME, concerns have existed about the absence of a universal long-term strategic plan with the need for repeated injections to maintain visual benefits, and the absence of knowledge of the long-term prognosis in subjects who are lost to follow-up or are discharged from the clinic. ${ }^{2}$ The cost of therapy ${ }^{3}$ and the continuous need for follow-up have encouraged investigators look for alternatives such as longer acting anti-VEGF agents, ${ }^{4}$ biosimilar drugs, ${ }^{5}$ and controlled release technologies. ${ }^{6}$ Because pan-retinal laser photocoagulation (PRP) has demonstrated long-term durability in the regression of proliferative diabetic retinopathy ${ }^{7}$ and some DME, ${ }^{8,9}$ we explored in a pilot study the effect of peripheral PRP
Correspondence: Ahmad M Mansour Department of Ophthalmology, American University of Beirut, PO Box I I 3-6044, Beirut, Lebanon

Tel +96I 3377633

Email ammansourmd@gmail.com 
(PPRP) on DME to answer the question of whether PPRP can decrease the number of anti-VEGF injections needed.

\section{Materials and methods}

The pilot prospective study received approval by the institutional review board of Rafik Hariri University Hospital and followed the tenets of the Declaration of Helsinki. The study was carried out from January 2015 to December 2018. Consecutive patients with naïve DME underwent spectral domain optical coherence tomography (OCT) and fluorescein angiography (FA) of the midperiphery and were offered to have continuous injections of anti-VEGF agents (treat and extend) if they were compliant, or PPRP with modified (flexible) pro re nata (PRN) injections if they felt unable to be followed frequently (bodily handicap; living abroad; long-distance travel; frequent hospitalizations; phobia of injections; and poor financial resources). The patient consent was both written and informed consent.

The injection of the drug ziv-aflibercept and regular OCT scans were made free of charge. In both groups, patients were treated at monthly intervals until maximal resolution of intraretinal edema by OCT. If, on follow-up recurrent intraretinal fluid was noted, monthly ziv-aflibercept treatment was resumed until stable OCT parameters were reached. The injection regimen (in the injection-only group) followed the protocol for DME which was 5 initial monthly injections then treat and extend by 2 weeks based on OCT. The injection regimen in the PPRP group was initial injection concomitant with the laser and then monthly recall for OCT or else whenever the patient can return at the earliest possible appointment (within 2 months period).

Ziv-aflibercept $0.05 \mathrm{~mL}$ (1.25 mg aflibercept) was prepared according to the standard compounding protocols and stored at $4{ }^{\circ} \mathrm{C}$ for 4 weeks. The same operator performed the vision examination, OCT test, and intravitreal injection. Best-corrected visual acuity (BCVA) was assessed by using Early Treatment Diabetic Retinopathy Study R chart (Precision Vision, La Salle, IL, USA). Central macular thickness (CMT) or mean thickness in central 1,000 $\mu \mathrm{m}$ diameter area was calculated (and also FA) using spectral domain OCT 3D-2000 Topcon FA plus (Topcon, Tokyo, Japan).

\section{Inclusion criteria}

The inclusion criteria were as follows: naïve DME cases; follow-up on each visit by same OCT machine; initial intravenous FA (with capture of the midperiphery); minimum follow-up of 18 months; recording of BCVA; and CMT after a washout of 2 months after the last injection.

\section{Exclusion criteria}

The exclusion criteria were as follows: type 1 diabetes mellitus, uncontrolled diabetes mellitus, uncontrolled systemic hypertension, renal failure, previous laser therapy, previous intraocular therapies (intravitreal corticosteroid drugs or implants), previous vitrectomy, proliferative diabetic retinopathy, severe preproliferative diabetic retinopathy, macular degeneration, vitreomacular traction, iris neovascularization, glaucoma, miotic pupil, corneal scar, blepharitis, and infectious conjunctivitis. As most patients had cataract, the follow-up was stopped when cataract surgery was performed.

\section{Technique of laser PRP}

After maximal pharmacological dilation of the pupil, argon green laser (Ellex ${ }^{\circledR}$ Laserex Integre 532 green retinal slit lamp laser; Ellex, Adelaide, Australia) was applied using $165^{\circ}$ wide angle lens (HR Wide Field ${ }^{\circledR}$; Volk Optical Inc., Mentor, OH, USA) and with minimal settings (200 $\mu$ spot size, $80 \mathrm{~ms}$ pulse duration, and $200-350 \mathrm{~mW}$ energy output) to achieve a mild grey-white burn $360^{\circ}$ from the equator to the extreme midperiphery concentrating on areas of ischemia depicted by FA. Ischemia was graded as mild (less than $25 \%$ of capillary nonperfusion at midperiphery) or moderate (if less than $50 \%$ capillary nonperfusion at midperiphery). ${ }^{10}$

\section{Results}

All study patients were Caucasians. There were 2 men and 1 woman (on wheelchair) in the PPRP group (3 right eyes and 3 left eyes) vs 3 men and 1 woman (4 right eyes) in the injection only group (Table 1). Mean age was 66.3 years in both groups. Mean duration of diabetes was 15.0 years in the PPRP group and 11.3 years in the injection only group.

Six eyes of 3 patients had PPRP versus 4 eyes of 4 patients had injections only. The number of anti-VEGF was markedly decreased when PPRP was administered from a mean number of injections of 16.5 (range 13-21; mean follow-up 24.3 months) in the injection only group to a mean number of 4.5 (range 0-8; mean follow-up 33.7 months) in the PPRP group. Mean number of OCT performed was 13.3 in the PPRP group and 20.8 in the injection only group. Mean number of laser spots was 1,364 (range 1,020-1,670). Mean initial CMT was $462.0 \mu$ in the injection only group vs $457.3 \mu$ in the PPRP group. Mean final CMT was $462.0 \mu$ in the injection only group vs $350.0 \mu$ in the PPRP group. Baseline and final mean $\log$ MAR (Snellen equivalent) BCVA were 0.84 (20/137) and $0.60(20 / 80)$ in the injection only group and 0.70 (20/100) and $0.69(20 / 98)$ in the PPRP group, respectively. Dense (grade 3 ) nuclear sclerotic cataract was present at the 
end of follow-up in all eyes in the PPRP group and in 2 eyes in the injection only group, with the remaining 2 eyes being pseudophakic. One eye developed transient mild anterior uveitis 2 days after the 13th ziv-aflibercept injection and was controlled with topical corticosteroids.

\section{Discussion}

The current preliminary study points to the possibility of minimizing the number of intravitreal ziv-aflibercept injections in DME when PPRP is performed. It could be argued that the PPRP group received less intravitreal injections than the injection only group (mean injection number 4.5 vs 16.5) because of longer intervals between OCT examinations (mean number of OCT scans per patient being $13.3 \mathrm{vs}$ 20.8) and shorter total mean follow-up time (20.8 months vs 33.7 months). However, OCT monitoring was comparable between the 2 groups: a mean of 6.6 OCT per year in the PPRP group vs 7.4 OCT for the injection only group.

The RISE and RIDE pivotal trials demonstrated sustained large visual improvements in eyes with DME when treated with ranibizumab. ${ }^{11}$ Although anti-VEGF therapy holds highest promise in the treatment of DME, the cost-benefit analysis and safety profile of monthly treatments likely indicate its role as the sole therapy in a majority of patients but there is a high rate of dropout. ${ }^{12,13}$ PRP is an effective long-term treatment for proliferative diabetic retinopathy with a relatively low side effect profile and significant cost efficacy in comparison to anti-VEGF therapy.

Although anti-VEGF therapy offers numerous advantages over PRP (better visual acuity), there are some limitations. Intravitreal injections are associated with a very low risk of endophthalmitis, retinal tear, cataract development, intraocular inflammation, worsening of vitreoretinal traction, and need for frequent follow-up and imaging. The financial burden of repeated intravitreal injections and imaging coupled with time off work, travel time or transportation costs, and partial visual recovery can pose a significant challenge to those with a history of poor compliance or with limited access to medical care. Potential side effects such as proteinuria, stroke, and myocardial infarction are life-threatening, and they limit our ability to use anti-VEGF in more systemically ill patients. ${ }^{14}$

PRP was found to be more cost-effective than intravitreal anti-VEGF injections in PDR, ${ }^{3}$ and this can be extrapolated to DME therapy as in the current combined PPRP group. ${ }^{3}$ PRP is often accompanied by a temporary drop in vision. Thirtyfour percentage of the 76 eyes with PDR and a normal macula showed morphological changes following PRP at 3 months. ${ }^{15}$ Intravitreal anti-VEGF before or concomitant with PRP can prevent post-laser exacerbation of DME. Suñer et al ${ }^{16}$ completed a pilot prospective randomized study on DME to assess the efficacy and durability of a single intravitreal ranibizumab with peripheral scatter laser to peripheral nonperfusion on ultrawide-field FA ( $\mathrm{n}=15)$ vs intravitreal triamcinolone acetonide plus macular laser $(n=15)$. At 6 months, the peripheral laser group had fewer recurrences of DME than the focal laser group warranting retreatment ( $33 \%$ vs $80 \%, p<0.003$ ) without significant difference in the mean change in visual acuity and central foveal thickness. In a study by Brown et $\mathrm{al}^{17}, 40$ eyes of 29 patients with DME were randomized in 1:1 ratio to $0.3 \mathrm{mg}$ ranibizumab or combination with targeted laser to ischemic retina guided by widefield FA. All eyes received 4 monthly ranibizumab injections followed by monthly examinations and PRN treatment for 36 months. There was no evidence that combination therapy with ranibizumab and targeted peripheral laser improved visual outcome or reduced treatment burden (mean number of injections of 24.4 in monotherapy vs 27.1 in ranibizumab and peripheral laser) compared with ranibizumab alone. Similar to these findings in DME, Spaide ${ }^{18}$ and Campochiaro et $\mathrm{al}^{19}$ did not find scatter laser to benefit macular edema in retinal vein occlusion on anti-VEGF therapy.

It is still unknown what happens when patients with DME on anti-VEGF therapy are lost to follow-up. Recent report ${ }^{2}$ raised the concerns about recurrence or rebound macular edema and progression to more advanced stages of diabetic retinopathy such as neovascular glaucoma and tractional retinal detachment from proliferative diabetic retinopathy. Eyes that received PPRP are protected against neovascular glaucoma and tractional retinal detachment, unlike eyes on intravitreal injections if they are lost to follow-up. Likewise, Obeid et $\mathrm{al}^{12}$ found that eyes with PDR that received only intravitreal anti-VEGF had worse anatomic and functional outcomes after being lost to follow-up than with eyes that received PRP. Similarly, we postulate that eyes that receive PPRP would end up with better outcomes when lost to followup than eyes on injections only.

The concept of combining single peripheral scatter laser (PPRP) to target the ischemic periphery and concomitantly inject anti-VEGF agents initially and $\mathrm{PRN}$ is to block preexisting and subsequent VEGF. The mechanisms of action of PRP in DME include induction of posterior vitreous detachment (Table 2) and ablation of ischemic peripheral retina where the reservoir of VEGF resides. ${ }^{20}$ If macular leakage is due to VEGF emanating from areas of peripheral retinal ischemia, then targeted laser to these sites should decrease DME and may diminish the number of VEGF inhibitor injections needed to control the disease (Table 2). Several studies detected 
Table I Clinical characteristics of the 2 groups of eyes having diabetic macular edema

\begin{tabular}{|l|l|l|l|l|l|l|l|l|l|l|}
\hline Groups & Case & Age & Race & Gender & $\begin{array}{l}\text { Duration } \\
\text { of } \\
\text { diabetes } \\
\text { (years) }\end{array}$ & Lipidemia & $\begin{array}{l}\text { Systemic } \\
\text { hypertension }\end{array}$ & $\begin{array}{l}\text { Site } \\
\text { Initial } \\
\text { vision } \\
\text { central } \\
\text { macular } \\
\text { thickness }\end{array}$ \\
\hline PPRP+inject & Case I & 70 & C & M & I5 & No & Yes & Left & $20 / 40$ & 559 \\
\hline PPRP+inject & Case I & 70 & C & M & I5 & No & Yes & Right & $20 / 200$ & 596 \\
\hline PPRP+inject & Case 2 & 6I & C & M & I8 & Yes & Yes & Left & $20 / 200$ & 564 \\
\hline PPRP+inject & Case 2 & 6I & C & M & I8 & Yes & Yes & Right & $20 / 200$ & 559 \\
\hline PPRP+inject & Case 3 & 68 & C & F & I2 & Yes & No & Right & $20 / 70$ & 202 \\
\hline PPRP+inject & Case 3 & 68 & C & F & I2 & Yes & No & Left & $20 / 70$ & 264 \\
\hline Inject only & Case 4 & 63 & C & M & 7 & No & No & Right & $20 / 200$ & 580 \\
\hline Inject only & Case 5 & 63 & C & M & 8 & No & No & Right & $20 / 125$ & 496 \\
\hline Inject only & Case 6 & 7I & C & F & I5 & No & Yes & Right & $20 / 200$ & 450 \\
\hline Inject only & Case 7 & 68 & C & M & I5 & No & Yes & Right & $20 / 70$ & 322 \\
\hline
\end{tabular}

Abbreviations: C, Caucasian; OCT, optical coherence tomography; PPRP, peripheral pan-retinal photocoagulation.

midperipheral ischemia in eyes with DME. ${ }^{21,22}$ Besides PPRP stops or halts progression from non-PDR to PDR and onset of neovascular glaucoma or rubeosis iridis. There is no doubt that intensive anti-VEGF protocols yield the best anatomic and visual outcomes; however, in noncompliant subjects, combining PPRP and injection may supposedly yield superior results than injection alone in the setting of poor compliance. Ideally to answer this proposition, there is a need to compare a noncompliant group receiving injections only to the current combination group. The main message is that skipping treatment in eyes with prior PPRP is not risky if we adapt the injection protocol to the special needs and circumstances of the particular patient. The modified PRN protocol here is to call the patient monthly and if not possible, let the patient be followed on his own pace with monthly reminder.

Table 2 Benefits and side effects of peripheral pan-retinal photocoagulation in diabetic macular edema

\begin{tabular}{|l|}
\hline Advantages \\
\hline Decrease VEGF secretion \\
\hline Decrease rubeosis iridis and neovascular glaucoma \\
\hline Blunt progression into proliferative diabetic retinopathy \\
\hline Decrease number of intravitreal anti-VEGF \\
\hline Induce PVD formation \\
\hline Cost-effective \\
\hline Suited for noncompliant subjects, very sick, and difficult to ambulate \\
\hline Ideal for patients with recent stroke or heart attack \\
\hline More durable effect \\
\hline Disadvantages \\
\hline Acute worsening of DME unless preceded by anti-VEGF injection \\
\hline Epiretinal membrane formation \\
\hline
\end{tabular}

Abbreviations: DME, diabetic macular edema; PVD, posterior vitreous detachment.
The monthly cost of the PPRP group was 3 times less than the injection only group assuming similar visual outcomes. This PPRP approach apparently is more cost-effective and patient-friendly than the more rigid and strict protocols relating to injections of anti-VEGF in DME.

The current pilot study has many limitations: absence of wide-field fluorescein imaging, small number of subjects, nonrandomized groups, nonmatching of clinical variables, short follow-up, and examiner bias. Moreover, the final visual acuity was less than expected in both groups because of worsening nuclear sclerosis during follow-up in both groups.

\section{Conclusion}

Although anti-VEGF monotherapy carries the best visual performance in the established protocols of DME, the costbenefit analysis and safety profile of monthly treatments likely indicate its role as an adjunct rather than the sole therapy. One session of PPRP with modified PRN injections is a potential effective long-term treatment for DME with a relatively low side effect profile and significant cost efficacy in comparison to anti-VEGF therapy alone. It is best suitable in noncompliant subjects with financial hardship and multiple health issues where discontinuation of injections may lead to irreversible visual loss. Further studies are needed to answer the question of how the standard anti-VEGF group does after being lost to follow-up versus the patients who had PPRP with less frequent intravitreal injections during therapy for DME.

\section{Disclosure}

JA received personal fees from Turing Pharmaceuticals LLC, personal fees from DORC International BV, personal fees 


\begin{tabular}{|c|c|c|c|c|c|c|c|c|c|}
\hline $\begin{array}{l}\text { Final } \\
\text { vision }\end{array}$ & $\begin{array}{l}\text { Final } \\
\text { central } \\
\text { macular } \\
\text { thickness }\end{array}$ & $\begin{array}{l}\text { Number of } \\
\text { intravitreal } \\
\text { injections }\end{array}$ & $\begin{array}{l}\text { Follow-up } \\
\text { (month) } \\
\text { after laser } \\
\text { or first } \\
\text { injection }\end{array}$ & $\begin{array}{l}\text { Retinal } \\
\text { ischemia }\end{array}$ & $\begin{array}{l}\text { Number } \\
\text { of laser } \\
\text { shots }\end{array}$ & $\begin{array}{l}\text { Dense cataract } \\
\text { at final } \\
\text { examination }\end{array}$ & $\begin{array}{l}\text { Number } \\
\text { of OCT }\end{array}$ & $\begin{array}{l}\text { Total } \\
\text { estimated } \\
\text { costs } \\
\text { (USD) }\end{array}$ & $\begin{array}{l}\text { Average } \\
\text { monthly } \\
\text { costs } \\
\text { (USD) }\end{array}$ \\
\hline $20 / 33$ & 255 & 8 & 31 & No & 1,670 & Yes & 17 & 3,500 & 113 \\
\hline $20 / 40$ & 201 & 5 & 42 & No & $\mathrm{I}, 020$ & Yes & 17 & 2,900 & 69 \\
\hline $20 / 200$ & 402 & 5 & 45 & No & $\mathrm{I}, 594$ & Yes & 13 & 2,500 & 56 \\
\hline $20 / 133$ & 532 & 6 & 42 & No & $\mathrm{I}, 500$ & Yes & 13 & 2,800 & 67 \\
\hline $20 / 200$ & 510 & 3 & 22 & Moderate & 1,200 & Yes & 10 & 1,600 & 73 \\
\hline $20 / 100$ & 200 & 0 & 20 & Moderate & 1,200 & Yes & 10 & 1,200 & 60 \\
\hline $20 / 50$ & 274 & 21 & 28 & Moderate & None & Yes & 26 & 6,800 & 227 \\
\hline $20 / 100$ & 569 & 13 & 23 & Mild & None & Yes & 17 & 4,300 & 187 \\
\hline $20 / 100$ & 447 & 18 & 27 & Mild & None & Pseudophakos & 21 & 5,700 & 211 \\
\hline $20 / 80$ & 240 & 14 & 19 & No & None & Pseudophakos & 19 & 4,700 & 247 \\
\hline
\end{tabular}

from Allergan Inc., personal fees from Bayer, personal fees from Mallinckrodt, and grants from TOPCON. The authors report no other conflicts of interest in this work.

\section{References}

1. Mitchell P, Bandello F, Schmidt-Erfurth U, et al. The RESTORE study: ranibizumab monotherapy or combined with laser versus laser monotherapy for diabetic macular edema. Ophthalmology. 2011;118(4): 615-625. doi:10.1016/j.ophtha.2011.01.031

2. Mookhtiar MA, Carrim ZI. Treating maculopathy at the expense of proliferative disease: an emerging problem in 'macular treatment centres'. Eye (Lond). 2014;28(11):1390-1391. doi:10.1038/eye.2014.160

3. Lin JS, Chang JS, Smiddy WE. Cost evaluation of panretinal photocoagulation versus intravitreal ranibizumab for proliferative diabetic retinopathy. Ophthalmology. 2016;123(9):1912-1918. doi:10.1016/j. ophtha.2016.05.037

4. Dugel PU, Jaffe GJ, Sallstig P, et al. Brolucizumab versus aflibercept in participants with neovascular age-related macular degeneration: a randomized trial. Ophthalmology. 2017;124(9):1296-1304. doi:10.1016/j. ophtha.2017.03.057

5. Sharma A, Reddy P, Kuppermann BD, Bandello F, Loewenstein A. Biosimilars in ophthalmology is there a big change on the horizon. Clin Ophthalmol. 2018;12:2137-2143. doi:10.2147/OPTH.S180393

6. Lau CML, Yu Y, Jahanmir G, Chau Y. Controlled release technology for anti-angiogenesis treatment of posterior eye diseases: current status and challenges. Adv Drug Deliv Rev. 2018;126:145-161. doi:10.1016/j. addr.2018.03.013

7. Chew EY, Ferris FL 3rd, Csaky KG, et al. The long-term effects of laser photocoagulation in patients with diabetic retinopathy. Ophthalmology. 2003;110(9):1683-1689. doi:10.1016/S0161-6420(03)00579-7

8. Gardner TW, Eller AW, Friberg TR. Reduction of severe macular edema in eyes with poor vision after panretinal photocoagulation for proliferative diabetic retinopathy. Graefes Arch Clin Exp Ophthalmol. 1991;229(4):323-328.

9. Gaucher D, Fortunato P, Le Cleire-Collet A, et al. Spontaneous resolution of macular edema after panretinal photocoagulation in florid proliferative diabetic retinopathy. Retina. 2009;29(9):1282-1288. doi:10.1097/IAE.0b013e3181a91e9f

10. Hamanaka T, Akabane N, Yajima T, Takahashi T, Tanabe A. Retinal ischemia and angle neovascularization in proliferative diabetic retinopathy. Am J Ophthalmol. 2001;132(5):648-658.
11. Brown DM, Nguyen QD, Marcus DM, et al. RIDE and RISE research group: long-term outcomes of ranibizumab therapy for diabetic macular edema: the 36-month results from two phase III trials: RISE and RIDE. Ophthalmology. 2013;120(10):2013-2022. doi:10.1016/j. ophtha.2013.02.034

12. Obeid A, Su D, Patel SN, et al. Outcomes of eyes lost to follow-up with proliferative diabetic retinopathy that received panretinal photocoagulation versus intravitreal anti-vascular endothelial growth factor. Ophthalmology. 2019;126(3):407-413. doi:10.1016/j.ophtha.2018.07.027

13. Griffin SJ. Lost to follow-up: the problem of defaulters from diabetes clinics. Diabet Med. 1998;15(Suppl 3):S14-S24. doi:10.1002/ (SICI)1096-9136(1998110)15:3+<S14:AID-DIA725>3.0.CO;2-I

14. Avery RL. What is the evidence for systemic effects of intravitreal anti-VEGF agents, and should we be concerned? Br J Ophthalmol. 2014;98:i7-i10. doi:10.1136/bjophthalmol-2013-303844

15. Soman M, Ganekal S, Nair U, Nair K. Effect of panretinal photocoagulation on macular morphology and thickness in eyes with proliferative diabetic retinopathy without clinically significant macular edema. Clin Ophthalmol (Auckland, NZ). 2012;(6):2013-2017.

16. Suñer IJ, Peden MC, Hammer ME, et al. A pilot study to assess the efficacy, durability, and safety of a single intervention with ranibizumab + peripheral laser for diabetic macular edema associated with peripheral nonperfusion on ultrawide-field angiography. Ophthalmologica. 2015;233(1):89-95.

17. Brown DM, Ou WC, Wong TP, Kim RY, Croft DE, WykoffCC. Targeted retinal photocoagulation for diabetic macular edema with peripheral retinal nonperfusion. Three-year randomized DAVE trial. Ophthalmology. 2018;125(5):683-690. doi:10.1016/j.ophtha.2017.11.026

18. Spaide RF. Prospective study of peripheral panretinal photocoagulation of areas of nonperfusion in central retinal vein occlusion. Retina. 2013; 33(1):56-62. doi:10.1097/IAE.0b013e3182641875

19. Campochiaro PA, Hafiz G, Mir TA, et al. Scatter photocoagulation does not reduce macular edema or treatment burden in patients with retinal vein occlusion. Ophthalmology. 2015;122(7):1426-1437. doi:10.1016/j. ophtha.2015.04.006

20. Mansour AM, Pulido JS, Arevalo JF. Diabetic macular edema: from old concepts to new therapeutic avenues. Med Hypothesis Discov Innov Ophthalmol. 2015;4(4):130-135.

21. Shimizu K, Kobayashi Y, Muraoka K. Midperipheral fundus involvement in diabetic retinopathy. Ophthalmology. 1981;88(7):601-612.

22. Sim DA, Keane PA, Rajendram R, et al. Patterns of peripheral retinal and central macula ischemia in diabetic retinopathy as evaluated by ultra-widefield fluorescein angiography. Am J Ophthalmol. 2014; 158(1):144-153. doi:10.1016/j.ajo.2014.03.009 


\section{Publish your work in this journal}

Clinical Ophthalmology is an international, peer-reviewed journal covering all subspecialties within ophthalmology. Key topics include: Optometry; Visual science; Pharmacology and drug therapy in eye diseases; Basic Sciences; Primary and Secondary eye care; Patient Safety and Quality of Care Improvements. This journal is indexed on

PubMed Central and CAS, and is the official journal of The Society of Clinical Ophthalmology (SCO). The manuscript management system is completely online and includes a very quick and fair peer-review system, which is all easy to use. Visit http://www.dovepress.com/ testimonials.php to read real quotes from published authors. 